\title{
Relationship Between Progression of Prostate Cancer and Walking
}

\author{
NOBUO OKUI* \\ *Yokosuka Urogynecology and Urology Clinic, Kanagawa, Japan
}

\begin{abstract}
Objective: Our facility has previously reported that periodic exercise, especially brisk walking with a monthly distance of approximately $120 \mathrm{~km}$, is healthy in men. In the present study, we examined the relationship between cancer-related mortality and prostate-specific antigen (PSA) values in prostate cancer patients by providing guidance concerning brisk walking, especially walking for a distance of $120 \mathrm{~km}$.

Materials and Methods: After diagnosis of prostate cancer, patients who were not indicated for operative treatment of the prostate cancer and selected combined androgen blockade (CAB) therapy also received guidance concerning brisk walking once monthly.

We examined the outdoor one-month walking distance of each patient once annually by pedometer. Exercise quantity, mortality rate, and serum PSA values were examined over an eight-year period.

Results: CAB therapy was initially initiated in 102 prostate cancer patients. Of the 48 persons who died during 8 years, mortality due to prostate cancer occurred in $41.6 \%$ (20 persons). There were significantly fewer prostate cancer-related deaths in the patient group that performed monthly exercise for $120 \mathrm{~km}$ or more than in the patient group that walked less than $120 \mathrm{~km}$.

Conclusions: The results of the present study demonstrate the possibility that exercise may lead to inhibition of prostate cancer and inhibition of PSA re-elevation. Actively introducing exercise into medical treatment may be effective for treating prostate cancer.
\end{abstract}

Key words: walking, prostate cancer, combined androgen blockade (CAB) therapy, mortality rate, prostate-specific antigen (PSA) value

\section{Introduction}

The number of prostate cancer patients has been increasing. In addition to surgery, treatment by endocrine therapy has been effective. In a system where cancer is amplified by androgen, treatment management using LH-RH antagonist or LH-RH agonist and an anti-androgen agent to lower androgen secretion from the testes is called $\mathrm{CAB}$ (combined androgen blockage) therapy.

However in recent years, a decrease in efficacy and progress of prostate cancer in cases of continued CAB therapy for several years has been observed. This is called castration-resistant prostate cancer (CRPC), and is a condition where long-term survival is not expected. Effective treatment methods for CRPC include new curative drugs such as Enzalutamide, Abiraterone, Cabazitaxel, but long-term observation of efficacy and side-effects is needed since these have just recently entered the market. Various opinions on the pathogenesis of CRPC have been reported, but even if $\mathrm{LH}-\mathrm{RH}$ antagonist or LH-RH agonist can control androgen secretion from the testes, the adrenal

\footnotetext{
Nobuo Okui

Yokosuka Urogynecology and Urology Clinic

The tower Yokosukachuo, LIDRE 3F

2-6 Ootakicho, Yokosuka-shi, Kanagawa 238-0008, Japan

TEL: +81-46-823-8456 E-mail: okui_nobuo@yahoo.co.jp

[Received Feb. 13, 2018] [Accepted Feb. 21, 2018〕
}

Copyright (C) 2018 The Juntendo Medical Society. This is an open access article distributed under the terms of Creative Commons Attribution License (CC BY), which permits unrestricted use, distribution, and reproduction in any medium, provided the original source is properly credited. doi: 10.14789/jmj.2018.64.JMJ18-P67 
gland still secretes androgen and efficacy on prostate cancer due to androgen has been determined to be the dominant cause ${ }^{1)}$. To support this, a recent report on patients receiving $\mathrm{CAB}$ therapy presented data that progression of cancer was observed in persons with high levels of circulating testosterone, which is representative of androgen, and progression could be controlled in those with lower levels ${ }^{2}$.

Consequently, an approach before conditions progress to CRPC is important. Activity in this field has attracted attention recently. In an article from the U.S., patients who frequently exercised and patients who did not were compared. Mortality rate of prostate cancer patients who did not exercise was set as 1 , and the rate for those who vigorously exercise three times a week was $0.39^{3)}$. Moreover, in another study, prostate progress risk of patients who comfortably walked less than 3 hours a week was set as 1 , and the rate for those who briskly walked more than 3 hours a week was 0.43 .

Unfortunately, there is no clear theory on the exercise effect mechanism ${ }^{4)}$. If exercise such as fast walking can be shown to be effective in controlling cancer in Japanese people, such knowledge can be used in the care of cancer patients.

In consideration of the above, this study focused on the distance a patient would leave the house and walk. Efficacy and the mechanism in Japanese people was considered by studying the effect of outdoor exercise on prostate cancer on total testosterone level, with or without CRPC, and change in PSA.

\section{Materials and Methods}

The subjects were 102 patients with prostate cancer (stage C or D) who selected hormone treatment from 2003 to 2008.

Brisk walking ${ }^{6)}$ was explained to all patients after onset of prostate cancer. Two Bimonthly brisk walking events were held to promote exercise among the prostate cancer patients. During the five-year period from 2003 to 2008, CAB therapy was initiated in 97 prostate cancer patients who did not undergo radical prostatectomy. During the monthly outpatient visits, we surveyed walking time and walking distance for each month. Once annually, we lent a pedometer (Omron pedometer
HJ-325-PK, etc.) to patients and measured the outdoor walking distance during a one-month period. We investigated whether mortality during the six-year observation period was due to prostate cancer or multiple diseases.

Blood sampling was annually performed in all patients at 9:00 in the morning of the day in which the pedometer was returned. Serum prostate-specific antigen (PSA) values were measured. Serum PSA values were measured by CLIA (unit: $\mathrm{ng} / \mathrm{ml}$, Shouwa Medical Science Corporation; normal value in healthy persons: $236-1,037 \mathrm{ng} / \mathrm{m} l$ ). Moreover, because the progression of prostate cancer has been reported to be inhibited in diabetic patients, we confirmed this in the patients.

In order to evaluate the general condition, performance status (PS) ${ }^{7)}$ was measured. One of the following scores was selected: 0: The patient has no problems in performing activities. The patient can perform activities of daily living without limitation in the same way as before onset. 1: Although physically intense activity is restricted, the patient is ambulatory and can perform light work and sitting work. 2: The patient is ambulatory and can perform all personal activities of daily living but can not work. The patient spends at least $50 \%$ of time outside of the bed during the daytime. 3: The patient is only able to perform limited personal activities of daily living. The patient spends at least $50 \%$ of time in bed or in a chair during the daytime. 4: The patient is completely unable to move. The patient is completely unable perform personal activities of daily living. The patient is completely restricted to a bed or chair.

Because we have previously found that the ideal monthly distance for middle-aged and elderly men is $120 \mathrm{~km}$, statistical investigation was carried out by dividing the patients into a group that performed outdoor brisk walking for a distance of $120 \mathrm{~km}$ or more and a group that walked less than $120 \mathrm{~km}^{8)}$.

Statistically significant differences were examined using student's T test. Satistical correlation was determined by calculating the Pearson correlation coefficient $r$, and when the absolute value of $\mathrm{r}(|\mathrm{r}|)$ was $0<|\mathrm{r}| \leq 0.2$, it was determined that there was almost no correlation. $0.2<|\mathrm{r}| \leq 0.4$ was defined as having a weak correlation. $0.4<|\mathrm{r}| \leq 0.7$ was defined as having a correlation. $0.7<|\mathrm{r}|<1.0$ 
was defined as having a strong correlation. 1.0 or -1.0 was defined as a complete correlation.

The present study was approved by the institutional review board of the Medical Association.

\section{Results}

At the start of 2003 to 2008, 102 prostate cancer patients were included and the mean age of subjects was 74.8 years ( \pm 4.9 years). Pathologic findings indicated that 45 patients had welldifferentiated adenocarcinoma, 55 had moderatelydifferentiated adenocarcinoma, and two had poorlydifferentiated adenocarcinoma. A total of 48 patients died during the eight-year observation period, and of these, mortality was due to prostate cancer in 20 persons (41.6\%).

First, we examined whether aging and $\mathrm{CAB}$ therapy lead to a decrease in in exercise capacity. Statistical examination of age and walking distance showed that there was no correlation using Pearson correlation coefficient (0.14). Based on these findings, in the present study, it appeared that the guidance of a physician brought about effects, and there was little correlation between age and walking distance. Because walking can be promoted to all patients regardless of age, we recommended walking for all patients. The duration of $\mathrm{CAB}$ therapy was 8.7 years $( \pm 0.8$ months). Statistical examination of the duration of $\mathrm{CAB}$ therapy and walking distance showed that the Pearson correlation coefficient was -0.01 . Based on these findings, the duration of $\mathrm{CAB}$ therapy and walking distance were not correlated in the present study.

Next, we investigated the group with a monthly walking distance of $120 \mathrm{~km}$ or more and group with a monthly walking distance less than $120 \mathrm{~km}$.

The PS and mortality numbers of the two groups were examined (Figure-1). The group with a monthly walking distance of $120 \mathrm{~km}$ or more comprised 51 persons; during the 8-year observation period, PS was 0 in all patients at time of performing measurements during urology department outpatient visit. There were 0 prostate cancer-related deaths and 16 total deaths during the observation period. By contrast, the group with a monthly walking distance less than $120 \mathrm{~km}$ comprised 51 persons, and PS was initially 0 in all patients, but while PS continued to be 0 in all

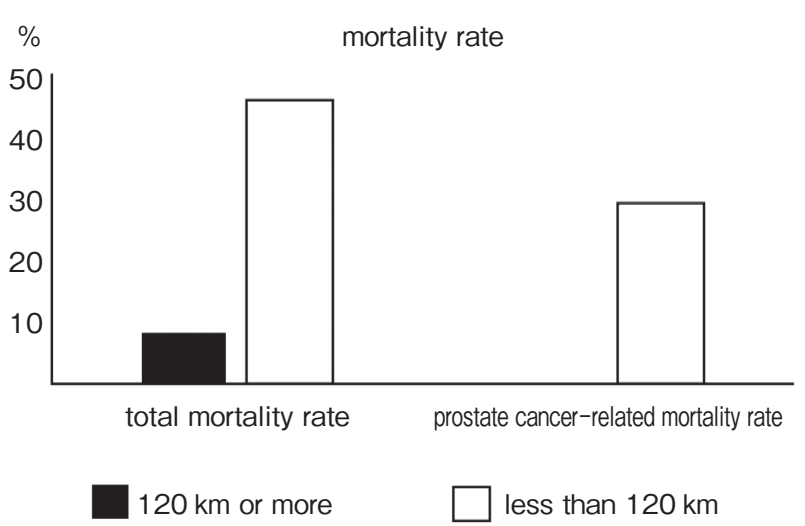

Figure-1

Graph comparing total mortality rate and prostate cancer-related mortality rate in the group that performed outdoor brisk walking for a distance of $120 \mathrm{~km}$ or more and the group that walked less than $120 \mathrm{~km}$. The black square indicates $120 \mathrm{~km}$ or more. The white square indicates less than $120 \mathrm{~km}$. Significant differences regarding both total mortality rate and prostate cancer-related mortality rate were noted between the groups $(\mathrm{p}<0.001)$.

patients during the first and second years of the observation period, PS was 2 in two patients in the third year. In the other patients, while PS was shown to be 0 , there were 16 patients with PS 1 and four patients with PS 2 in the sixth year. Moreover, in this group, there were 20 prostate cancer-related deaths and 32 deaths in total. Of the prostate cancer-related deaths in this group, 20 patients had PS of 3 before death. No patients had PS of 4 .

Next, the significant differences between the two groups were investigated. As shown by the Kaplan-Meier survival curves of the two groups in Figure-2, there was a significant difference in the total number of deaths in the group with a monthly walking distance of $120 \mathrm{~km}$ or more and group with a monthly walking distance less than $120 \mathrm{~km}$ ( $\mathrm{p}<$ 0.01 ). Furthermore, when cases of mortality due to other diseases are excluded, the group with a monthly walking distance of $120 \mathrm{~km}$ or more comprised 35 persons and group with a monthly walking distance less than $120 \mathrm{~km}$ comprised 39 persons, and a significant difference in the number of cases of prostate cancer-related deaths between the two groups was noted $(\mathrm{p}<0.01)$.

The relationship with the pathologic results of prostate cancer in the two groups was investigated. Of the patients who died due to other diseases in the group with a monthly walking distance of $120 \mathrm{~km}$ or more, eight had well-differentiated adenocarcinoma and eight had moderately-differentiated 

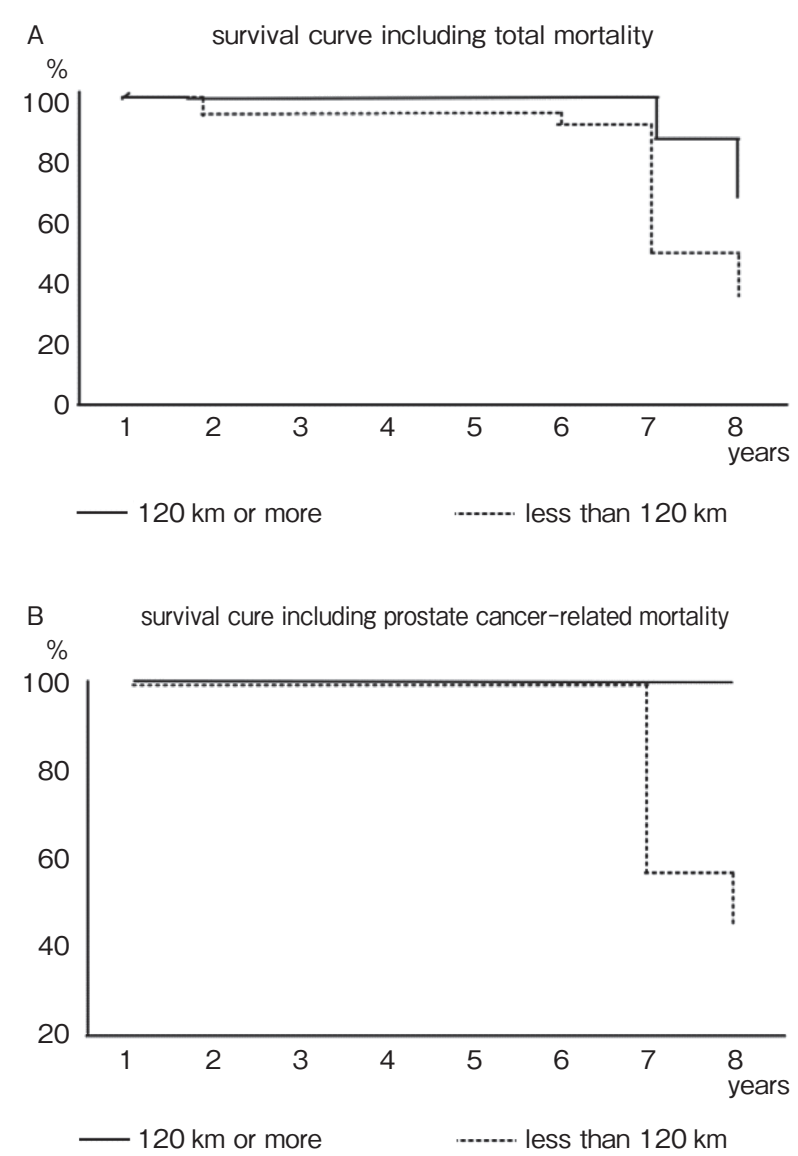

Figure-2

Changes over time of survival curve (A) including total mortality of two groups by length of monthly brisk walking $(20 \mathrm{~km}$ or more and less than $120 \mathrm{~km}$ ) and survival curve of prostate cancerrelated mortality only (B). Both show that the solid line is a group of $120 \mathrm{~km}$ or more. The dotted line shows the group of less than $120 \mathrm{~km}$. Significant differences between the groups for both mortality rates were noted $(\mathrm{p}<0.001)$.

adenocarcinoma. In the group with a monthly walking distance less than $120 \mathrm{~km}$, prostate cancerrelated deaths (total deaths) were as follows: patients with well-differentiated adenocarcinoma: 0 persons (4 persons), patients with moderatelydifferentiated adenocarcinoma: 18 persons (26 persons), and patients with poorly-differentiated adenocarcinoma: two persons (two persons). Early $\mathrm{CAB}$ therapy had no effects in patients with poorly-differentiated adenocarcinoma. Two patients with a PS of 2 in the third year had poorlydifferentiated adenocarcinoma.

The relationship with PSA values in the two groups was examined. In the group with a monthly walking distance of $120 \mathrm{~km}$ or more, PSA value was $83.6 \mathrm{ng} / \mathrm{m} l( \pm 34.7 \mathrm{ng} / \mathrm{m} l)$ before treatment and $0.084 \mathrm{ng} / \mathrm{m} l( \pm 0.1 \mathrm{ng} / \mathrm{m} l)$ at one year after $\mathrm{CAB}$

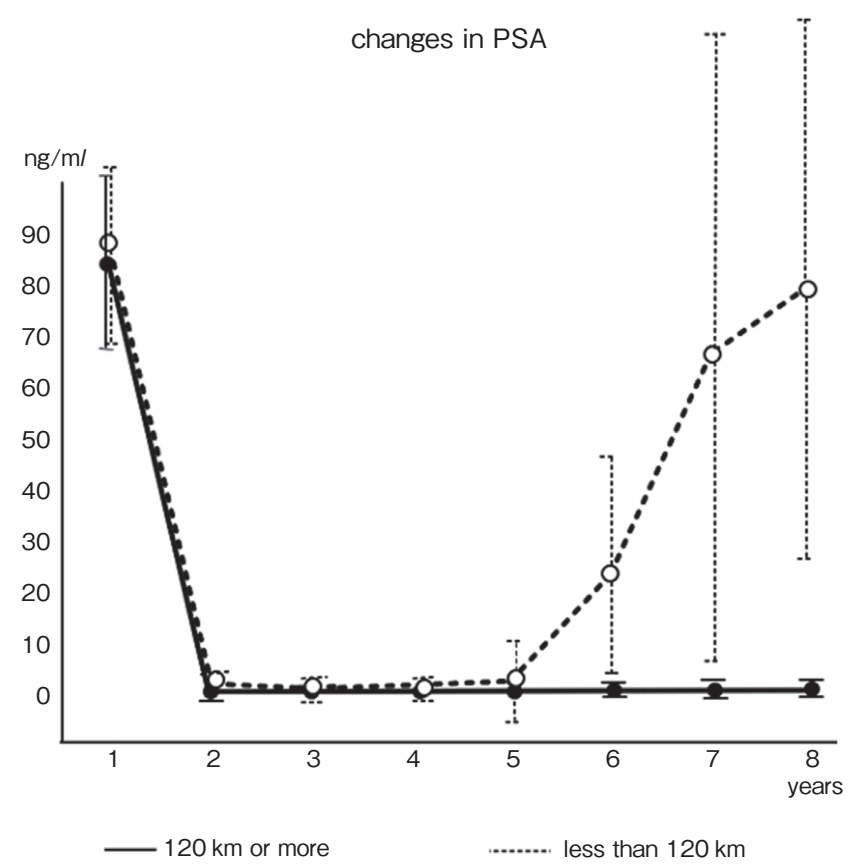

Figure-3

Graph showing changes in PSA The vertical axis shows PSA (unit: $\mathrm{ng} / \mathrm{d} l$ ), and horizontal axis shows number of years. The solid line and black circles indicate the group of $120 \mathrm{~km}$ or more per month. The dotted line and white circles indicate the group of less than $120 \mathrm{~km}$ per month. Significant differences were noted between the groups $(\mathrm{p}<0.001)$.

therapy; the mean value after the eight-year observation was $0.23 \mathrm{ng} / \mathrm{m} l( \pm 0.29 \mathrm{ng} / \mathrm{m} l)$. In the group with a monthly walking distance less than $120 \mathrm{~km}$, when the two patients with poorly-differentiated adenocarcinoma two died during the second year are excluded, PSA value was $87.3 \mathrm{ng} / \mathrm{m} l$ $( \pm 48.3 \mathrm{ng} / \mathrm{m} l)$ before treatment and $0.91 \mathrm{ng} / \mathrm{m} l$ $( \pm 0.9 \mathrm{ng} / \mathrm{m} l)$ at one year after CAB therapy; mean value after the eight-year observation was $57.0 \mathrm{ng} / \mathrm{m} l( \pm 80.1 \mathrm{ng} / \mathrm{m} l)$. Figure -3 shows the changes in PSA in the two groups, with the two patients with poorly-differentiated adenocarcinomas that showed significantly different PS values excluded. Based on these findings, it can be said that there was a significant difference in the PSA values of the two groups $(p<0.01)$. The values change along with the progress of prostate cancer, which is highly significant.

\section{Discussion}

Due to the increase in the number of prostate cancer patients ${ }^{1}$, inhibition of the disease is a major issue in Japan. It is well known in literature that the 
progress of cancer is inhibited by exercise ${ }^{2)-5)}$, and it would be of great significance if this also applied to Japanese persons.

Research has shown that if prostate cancer patients are able to maintain a monthly walking distance of $120 \mathrm{~km}$, the risk of prostate cancerrelated mortality is low. However, within the scope of the present study, poorly-differentiated adenocarcinoma carcinomas that show rapid clinical progression are included, and it is difficult to say that exercise alone has a inhibitory effect on prostate cancer. In fact, PS worsened early on in two patients with poorly-differentiated adenocarcinoma. However, based on these findings, there was a large difference in the effects of exercise. Furthermore, based on serum PSA values, it can be seen that the more often exercise is performed, the more prostate cancer is inhibited.

It is possible that total testosterone value may be related to the reason for the inhibitory effects of physical activity on prostate cancer. Testosterone is secreted from the testes and the adrenal gland and is actively transported to cells with androgen receptors (AR), where it intracellularly binds to AR. Therefore, in cases of prostate cancer, in which a large quantity of AR is produced, develops when total testosterone level is high, and tends to lead to prostate cancer-related mortality. For this reason, in the field of urology, as CAB therapy, treatment using LH-RH analogue or LH-RH antagonist is used to lower total testosterone level. Our findings have shown that regarding physical activity, when long-distance exercise is performed every month, the level is low. That is, it is conceivable that the total testosterone level of prostate cancer patients is lowered by physical activity and this forms some mechanism of delaying the progression of prostate cancer.

The involvement of diabetes in persons with low physical activity and low total testosterone is noteworthy. There is a relationship between diabetes and decreased exercise capacity, so walking distance is small. It is well known that diabetic patients have decreased testosterone production due to insulin resistance, and it has also been reported that because sex hormone-binding globulin is decreased, prostate cancer is inhibited.

In promoting exercise in prostate cancer patients, it is important that all departments related to geriatric medicine collaborate to provide guidance concerning exercise. Collaboration between orthopedic and internal medicine departments is especially important. The involvement of orthopedic department is useful in providing care through exercise, and for treating diabetes, collaboration with the internal medicine department is essential with respect to hypoglycemia during exercise, hypertension, cerebral infarction, myocardial infarction, cardiac rehabilitation, etc. Particularly, as the Tokyo Olympic Games will be held in Japan and the motivation for exercise increases throughout the country, knowledge of the possibility of inhibition of prostate cancer by exercising may greatly change the awareness of patients and force them to exercise more actively.

We examined the degree of a monthly walking distance of $120 \mathrm{~km} /$ month. The stride length in elderly persons performing brisk walking ranges from approximately 60 to $70 \mathrm{~cm}$, and approximately 8,000 steps walked in one day is equivalent to walking approximately $6 \mathrm{~km}$. If exercise is performed 20 days per month, this totals approximately $120 \mathrm{~km}$. Walking is effective even if the walking session are divided into twice per day. In the present study, the exercise intensity of walking was not specified, but it is the authors' opinion that favorable results were obtained. Therefore, it is possible to instruct patients to perform walking over $120 \mathrm{~km}$ per month without any strain.

Finally, the limitation of the present study is that the subjects were limited to patients who were treated by the author as their attending physician. Providing awareness concerning exercise to prostate cancer patients by the attending physician is substantial and has an effect on many of the patients. Therefore, although elderly persons seem not to walk much, they are characterized by the ability to make efforts concerning exercise and walk a sufficient distance. That is, the personality of the physician creates a bias, and obtained data cannot necessarily be said universal in Japan. It is the authors' opinion that this can be solved by conducting a large-scale investigation including multiple facilities.

\section{Conclusion}

Inhibition of prostate cancer was noted in 
patients who performed ongoing exercise. Furthermore, there were few cases of castration-resistant prostate cancer. These findings suggest that exercise may be effective in treating prostate cancer.

\section{References}

1) Ryan CJ, Tindall DJ: Androgen receptor rediscovered: the new biology and targeting the androgen receptor therapeutically. J Clin Oncol, 2011; 29: 3651-3658.

2) Klotz L, O'Callaghan C, Ding K, et al: Nadir testosterone within first year of androgen-deprivation therapy (ADT) predicts for time to castration-resistant progression: a secondary analysis of the PR-7 trial of intermittent versus continuous ADT. J Clin Oncol, 2015; 33: 1151-1156.

3) Kenfield SA, Stampfer MJ, Giovannucci E, Chan JM: Physical activity and survival after prostate cancer diagnosis in the health professionals follow-up study. J Clin Oncol, 2011; 29: 726-732.

4) Richman EL, Kenfield SA, Stampfer MJ, et al: Physical activity after diagnosis and risk of prostate cancer progression: data from the cancer of the prostate strategic urologic research endeavor. Cancer Res, 2011; 71: 3889-3895.

5) Friedenreich CM, Wang Q, Neilson HK, Kopciuk KA, McGregor SE, Courneya KS: Physical activity and survival after prostate cancer. Eur Urol, 2016; 70: 576-585.

6) Nobuo Okui: Jinsei wo kaeru 15 fun hayaaruki. Tokyo: Baseball Magazin Press, 2015.

7) National Cancer Institute - Common Toxicity Criteria (NCI-CTC Version 2.0, April 30, 1999). http://www. jcog.jp/doctor/tool/C_150_0011.pdf

8) Nobuo Okui: The study of testosterone and sexual function as a marker of health citizen sports. The Japanese Journal of Sexual Medicine, 2017; 32: 27-34. (in Japanese) 